\title{
High frequency of polyoma BK virus shedding in the gastrointestinal tract after hematopoietic stem cell transplantation: a prospective and quantitative analysis
}

\author{
ASY Wong ${ }^{1}$, VCC Cheng ${ }^{2}$, K-Y Yuen ${ }^{2}$, Y-L Kwong ${ }^{1}$ and AYH Leung ${ }^{1}$ \\ ${ }^{1}$ Department of Medicine, University of Hong Kong, Hongkong and ${ }^{2}$ Department of Microbiology, University of Hong Kong, \\ Hongkong
}

The polyoma BK virus (BKV) remains latent after primary infection and may reactivate during immunosuppression. The uroepithelium is the main latency site defined. This study addressed whether the gastrointestinal tract might be another latency site. To test this hypothesis, we prospectively quantified fecal BKV by quantitative PCR reaction in 40 patients undergoing hematopoietic SCT (HSCT). Urinary BKV was similarly quantified. Fecal BKV excretion was positive in 16/40 patients, of whom 10 were transient $(<3$ consecutively positive samples), six were persistent ( $\geqslant 3$ consecutively positive samples) and three were persistent with peaking $\left(\geqslant 10^{3}\right.$-fold increase in viral load over baseline, reaching $5.11 \times 10^{6}, 4.68 \times 10^{7}$ and $2.75 \times 10^{8}$ copies/sample at 14 , 14 and 21 days post-HSCT, respectively). Urinary BKV excretion was positive in $25 / 40$ patients. Fecal BKV excretion was significantly correlated with that of the urine $(P=0.036)$ and was significantly associated with allogeneic HSCT $(P=0.037)$ and persistent and peaking of urinary $\mathrm{BKV}$ excretion $(P<\mathbf{0 . 0 0 1})$. Binary logistic regression showed that $B K V$ viruria was the only significant risk factor for fecal BKV excretion $(P=0.021)$. Fecal BKV excretion occurred in $40 \%$ patients undergoing HSCT, implicating the gastrointestinal tract as a BKV latency site.

Bone Marrow Transplantation (2009) 43, 43-47; doi:10.1038/bmt.2008.266; published online 6 October 2008 Keywords: polyoma BK virus; hematopoietic SCT; gastrointestinal tract

\section{Introduction}

Polyoma BK virus (BKV) is a nonenveloped DNA virus containing a 5-kb circular double-stranded DNA. ${ }^{1}$ Primary infection occurs during childhood and is largely asymptomatic. The virus subsequently remains latent, and may undergo reactivation during immunosuppression.

Two distinct diseases due to $\mathrm{BKV}$ reactivation are recognized: polyomavirus-associated nephropathy in renal allografting and severe postengraftment hemorrhagic cystitis (HC) in hematopoietic SCT (HSCT). ${ }^{2-3}$ As both diseases involve the urinary tract, the uroepithelium has been proposed to be the main site of BKV latency.

Recently, data from another closely related polyoma virus, JC virus, showed that the gastrointestinal tract may be a latency site. ${ }^{4}$ Interestingly, BKV has also been detected by PCR in the stool of about $40 \%$ of hospitalized children. ${ }^{5}$ Nonetheless, these data collected at single time points did not distinguish primary infection from reactivation of latent infection. Moreover, quantification of viral load was lacking, precluding kinetic analysis of the viral reactivation process. As a result, it remains unclear whether the gastrointestinal tract could be a latency site for BKV.

In this study, we hypothesized that if the gastrointestinal tract was a site of $\mathrm{BKV}$ latency, viral reactivation might occur there during immunosuppression. To test this proposition, patient populations with frequent $\mathrm{BKV}$ reactivation would need to be studied. Taking advantage of the high incidence of $\mathrm{BKV}$ reactivation after $\mathrm{HSCT}$, we quantified prospectively the fecal BKV load in consecutive HSCT patients. Urine BKV load was quantified in parallel to indicate the possible concomitant uroepithelial viral reactivation.

\section{Materials and methods}

\section{Patients and HSCT protocols}

A consecutive unselected cohort of 40 patients undergoing HSCT at Queen Mary Hospital, Hong Kong, was studied. The transplantation protocol, antimicrobial prophylaxis and prevention and treatment of GVHD were also
Correspondence: Dr YL Kwong or Dr AYH Leung, University Department of Medicine, Professorial Block, Queen Mary Hospital, Pokfulam Road, Hong Kong.

E-mails: ylkwong@hkucc.hku.hk or ayhleung@hkucc.hku.hk

Received 4 February 2008; revised 17 July 2008; accepted 22 July 2008; published online 6 October 2008 
described. ${ }^{6}$ The informed consent was obtained from the patients and the investigation was approved by the institution review board in accordance with the Declaration of Helsinki.

\section{Stool sample collection}

Stool samples were collected prospectively before conditioning, on the day of HSC infusion, and weekly thereafter until day 50 or discharge. Methods of stool collection for viral studies have been reported. ${ }^{7}$ Briefly, stool was passed into a bedpan and from the center, approximately $0.5 \mathrm{~g}$ of stool was collected and swirled into $5 \mu$ of viral transport medium. DNA was extracted from an aliquot of $200 \mu \mathrm{l}$ of stool suspension (QIAamp Blood Minikit, Qiagen, Basel, Switzerland) and eluted with $200 \mu$ l of buffer.

\section{Urinary sample collection}

Serial spot urine samples $(50 \mathrm{ml})$ were collected at weekly intervals as aforementioned. The sample was spun at $2000 \mathrm{~g}$ for $10 \mathrm{~min}$. DNA was extracted from $200 \mu \mathrm{l}$ of free urine (QIAamp Blood Minikit) and eluted with $200 \mu$ l of buffer.

\section{BKV quantification}

Quantification of BKV from fecal and urinary samples was performed by Q-PCR with the ABI Prism 7700 Sequence Detector (PE Biosystems, Foster City, CA, USA). Sequences of the Q-PCR primers and TaqMan probe (targeting the BKV VPl gene), amplification protocols,

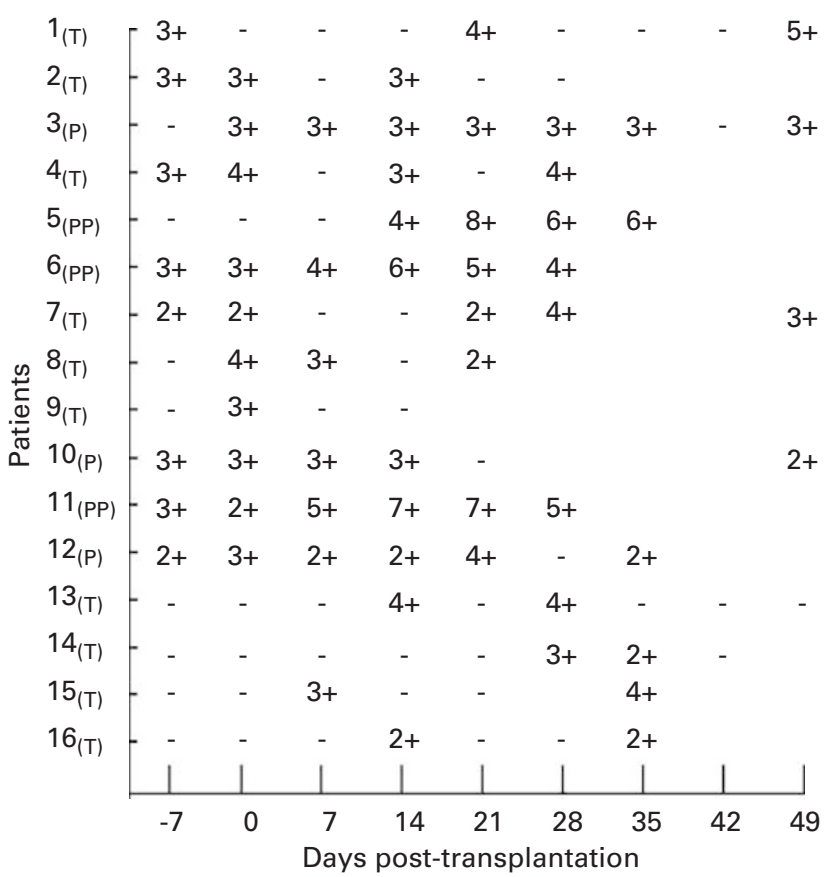

Figure 1 Fecal BK virus (BKV) excretion in 15 patients undergoing hematopoietic SCT (HSCT). The number at each time point represented the logarithm of the BKV copies/stool sample $(\sim 0.5 \mathrm{~g}$ of fecal material). For example, $3+$ represented $10^{3}$ copies and $5+$ represented $10^{5}$ copies $(-)$ denoted negativity for BKV. T $=$ transiently positive $(<3$ consecutive positive samples; note that there might be $\geqslant$ nonconsecutive positive samples); $\mathrm{P}=$ persistently positive ( $\geqslant 3$ consecutive positive samples); $\mathrm{PP}=$ persistent positive with peaking ( $\geqslant 3$-log increase over baseline).
PCR precautions and quality assurance have been described. ${ }^{6}$ All samples were tested in triplicates. Viral excretion was expressed in BKV genome copies per fecal sample $(0.5 \mathrm{~g}$ stool $)$ or per milliliter of urine.

\section{Controls for PCR interference}

For stool samples found negative for BKV by Q-PCR, fresh aliquots were obtained from the original stock stool suspensions. Known amounts of the BKV VPl plasmid standard ${ }^{6}$ were spiked into them. DNA was then extracted from these samples and Q-PCR performed. In all experiments, negative stool samples spiked with control BKV VPI plasmid showed Q-PCR results corresponding to the amount of input BKV plasmid, thereby excluding false negatives due to PCR failure arising from possible nonspecific interference in the stool (data not shown).

\section{Types of BKV excretion}

For this study, stringent criteria were used. BKV excretion, both fecal and urinary was defined as positive only if two or more samples were positive. Furthermore, we surmised that three or more consecutively positive samples (representing a continuous duration of 3 or more weeks) might be more biologically relevant. Therefore, positive samples were defined as transient when positive for $<3$ consecutive samples, and persistent when positive for $\geqslant 3$ consecutive samples. Persistent positive samples with significant peaking were defined as a peak BKV viral load with $\mathrm{a} \geqslant 10^{3}$-fold increase over baseline. ${ }^{6,8}$

\section{Statistical analysis}

Comparisons between groups of numerical data were evaluated by Kruskal-Wallis, and categoric data by $\chi^{2}$-tests. Risk factors for fecal BKV excretion were evaluated by binary logistic regression (SPSS, Chicago, IL, USA). The occurrence of fecal BKV reactivation was the dependent variable, with age, gender, types of HSCT (autologous vs sibling donors vs matched unrelated donors), sources of HSC (BM vs peripheral blood), conditioning regimens, occurrence of acute GVHD and $\mathrm{HC}$, and the types of urinary $\mathrm{BKV}$ reactivation as covariates. $P$-values of $<0.05$ were considered statistically significant.

\section{Results}

Samples

A total of 283 fecal and 342 urinary samples were quantified for BKV DNA (referred herewith BKV for short, median number of samples evaluated per patient: fecal $=8.0$; urine $=9.0$ ).

\section{Fecal BKV excretion}

Fecal BKV excretion was detectable in 16 patients $(40 \%)$ (transient, $n=10$; persistent, $n=3$; persistent with peaking, $n=3$ ) (Figure 1). Interestingly, in eight patients, fecal BKV was already detectable before marrow conditioning, although at low levels $\left(10^{2}-10^{3}\right.$ copies/stool sample). While 
the fecal BKV load might fluctuate in patients with persistent excretion, three patients showed significant peaking, reaching $5.11 \times 10^{6}, 4.68 \times 10^{7}$ and $2.75 \times 10^{8}$ copies/sample at 14, 14 and 21 days post-HSCT respectively, before declining to baseline levels. Fecal BKV was negative in the other $24 / 40$ patients.

\section{Urinary $B K V$ excretion}

Urinary BKV excretion was detectable in 25 patients $(65.0 \%)$ (transient, $n=4$; persistent, $n=2$; persistent with peaking, $n=19)$.

\section{Patterns of fecal and urinary BKV excretion}

Three different patterns of fecal and urinary BKV excretion could be distinguished (Figure 2). Pattern a patients $(n=3)$ showed concomitant and parallel increases and declines of fecal and urinary BKV load, with peaking of fecal and urinary BKV excretion coinciding temporally (fecal:
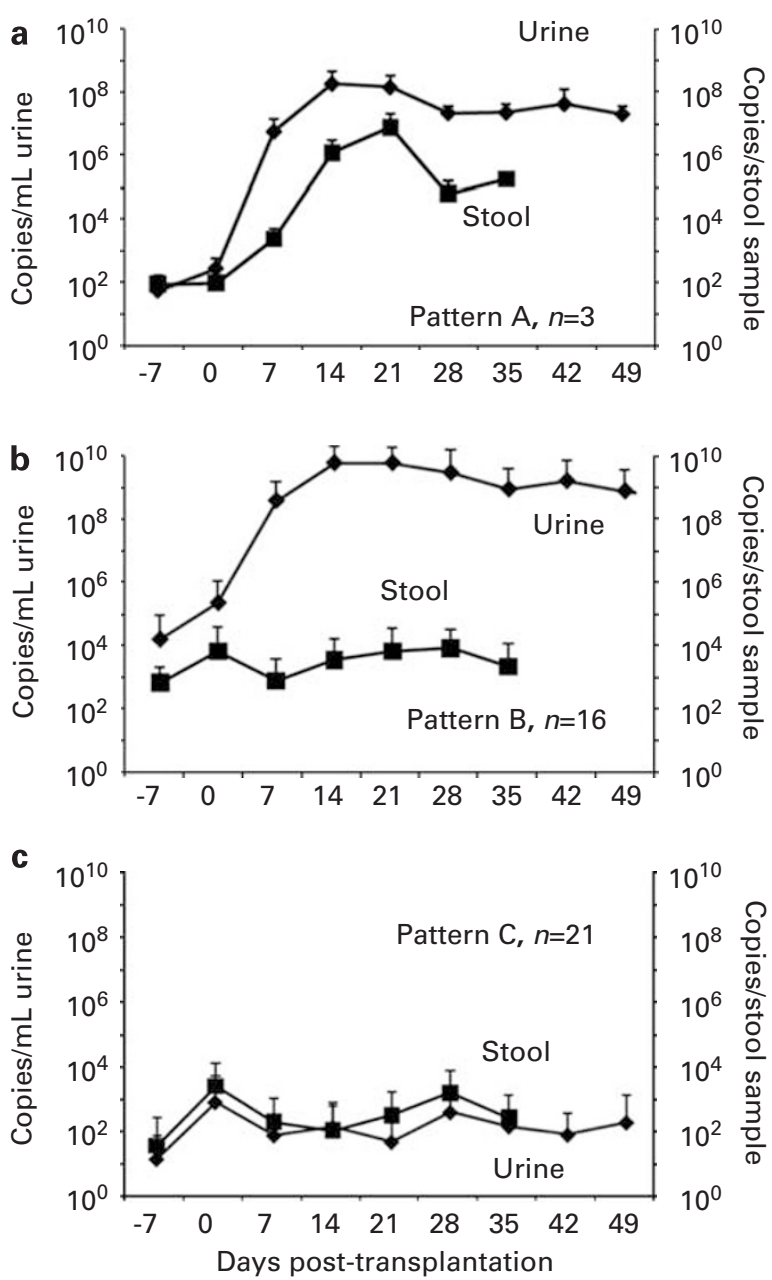

Figure 2 Patterns of fecal and urinary BKV excretion. (a) Three patients developed concomitant fecal and urinary BKV peaking. (b) Sixteen patients developed urinary but not fecal BKV peaking. (c) Twenty-one patients developed neither urinary nor fecal BKV peaking. Each data point represented mean results of BKV excretion at each time point for all the patients in that group. Therefore, although some patients in patterns $b$ and c never had quantifiable fecal BKV, the stool line did not touch baseline. Each error bar represented one s.d. BKV, BK virus.
$5.11 \times 10^{6}$ copies/sample, urinary: $5.47 \times 10^{9}$ copies $/ \mathrm{ml}$, day 14 ; fecal: $4.68 \times 10^{7}$ copies/sample, urinary: $4.67 \times 10^{8}$ copies/ml, day 14 ; fecal: $2.75 \times 10^{8}$ copies/sample, urinary: $1.54 \times 10^{9}$ copies $/ \mathrm{ml}$, day 21$)$. All three patients underwent allogeneic HSCT, each having significant GVHD. The first patient, who received HSCT from HLA-identical sibling, developed postengraftment $\mathrm{HC}$ with gross hematuria and dysuria on day 42 , and subsequent liver and skin GVHD at 6 months. The second patient, who received HSCT from matched unrelated donor, developed postengraftment $\mathrm{HC}$ on day 35. The third patient received HSCT from HLAidentical sibling and developed acute skin GVHD on day 26. Pattern b patients $(n=16)$ showed significant peaking of urinary BKV excretion, at a median of $2.28 \times 10^{8}$ $\left(6.33 \times 10^{4}-3.09 \times 10^{9}\right)$ copies $/ \mathrm{ml}$, occurring at a median of 24.5 (14-56) days after HSCT. However, none of these patients had significant peaking in fecal BKV excretion. Eleven patients received HSCT from HLA-identical siblings and two from matched unrelated donors. Three patients received autologous HSCT. In these patients, the types of fecal BKV excretion were persistent in two cases, transient in eight cases and negative in six cases. Pattern $\mathrm{c}$ patients $(n=21)$ showed no detectable peaking in urinary BKV excretion. Eleven patients received HSCT from HLAidentical siblings and three from matched unrelated donors. Seven patients received autologous HSCT. In these patients, fecal BKV excretion was persistent in one, transient in two, and negative in 18 patients.

\section{Correlation between fecal and urinary $B K V$ excretion}

The types (negative, transient, persistent and persistent with peaking) of urinary and fecal BKV excretion were significantly correlated (Kendall's Tau-b correlation coefficient $0.488, P=<0.01$; Figure 3 ). It was noteworthy that all but one patient with positive fecal BKV excretion have had urinary $\mathrm{BKV}$ excretion. On the other hand, 10 patients with positive urinary BKV excretion, including six patients with viral peaking, showed no fecal BKV (encircled points, Figure 3).

Clinicopathologic correlation of fecal BKV excretion To examine the risk factors and clinical correlation of fecal BKV excretion, the clinicopathologic parameters of patients with or without fecal BKV excretion were compared (Table 1). Positive stool BKV excretion was significantly associated with allogeneic HSCT $(P=0.037)$ as well as persistent and peaking of urinary BKV excretion $(P<0.001)$. Binary logistic regression showed that BKV viruria was the only significant risk factor for fecal $\mathrm{BKV}$ excretion $(P=0.021$, odds ratio: $0.101,95 \%$ confidence interval: $0.015-0.705)$.

\section{Discussion}

Although BKV reactivation has been described in solid organ allografting, mostly in renal transplantation ${ }^{9}$ and occasionally in liver and heart/lung transplantation, ${ }^{10}$ the actual frequency of reactivation is lower than that in HSCT, because of the much more intense immunosuppres- 


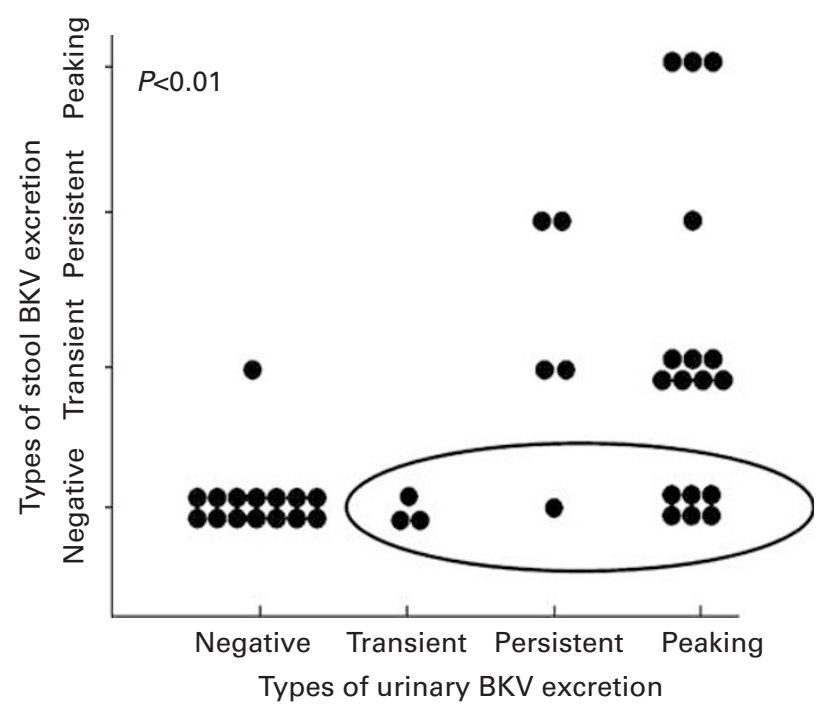

Figure 3 Correlation between fecal and urinary BKV excretion. The patterns were divided into transient $(<3$ consecutively positive samples during HSCT), persistent ( $\geqslant 3$ consecutively positive samples) and persistent with peaking ( $\geqslant 10^{3}$-fold increase from baseline). Each point represented data from an individual patient. The types of BKV excretion in fecal and urinary samples were significantly correlated (Kendall's Tau-b correlation coefficient $0.488, P<0.01$ ). The encircled points denoted 10 patients with positive BKV viruria, but no detectable fecal BKV excretion. BKV, BK virus; HSCT, hematopoietic SCT.

sion involved in the latter. ${ }^{3,6,8,11}$ Therefore, HSCT represents a unique opportunity to address the issue of whether BKV might be latent in the gastrointestinal tract, by studying fecal BKV load.

This is the first prospective and quantitative analysis of fecal BKV load during HSCT. We showed positive fecal BKV excretion in 16 of 40 HSCT patients. Our protocol of fecal viral quantification has been validated for coronavirus associated with severe acute respiratory syndrome. ${ }^{7}$ A significant peaking in fecal BKV excretion was detected in 3/40 patients (pattern a cases), which showed a consistent pattern of increase, peaking and decline. It paralleled concomitant urinary $\mathrm{BKV}$ reactivation, with peaks coinciding on the same days. In fact, all three patients had undergone allogeneic HSCT and developed GVHD, which were typical risk factors for urinary BKV reactivation. ${ }^{3,6,8}$ Therefore, fecal and urinary BKV excretions probably reflected viral reactivation consequent to severe immunosuppression. Definitive proof of BKV reactivation from the gastrointestinal tract would require detection of $\mathrm{BKV}$ viral antigen in colonic tissues, direct demonstration of viral particles in stool by electron microscopy or detection of BKV DNA in the gastrointestinal tract by in situ hybridization in the absence of late gene expression in seropositive patients. These issues warrant further investigations.

Previous studies of isolated colonic biopsies ${ }^{12}$ or ad hoc stool specimens showed the detection of BKV in the gastrointestinal tract. ${ }^{5}$ As these studies examined specimens at single time points, the actual kinetics, ${ }^{13}$ and the significance of the presence of BKV remain obscure. Our findings offered much more rigorous results and provided kinetic data that were more meaningful and robust that isolated measurements. Indeed, the increase and decline of
Table 1 Correlation between clinicopathologic characteristics and fecal BKV excretion

\begin{tabular}{|c|c|c|c|}
\hline & \multicolumn{2}{|c|}{ Fecal BKV excretion } & \multirow[t]{2}{*}{$\mathrm{P}$-value } \\
\hline & Positive & Negative & \\
\hline Number & 16 & 24 & \\
\hline Male:Female & $9: 7$ & $18: 6$ & 0.305 \\
\hline Age (years) & $42.4 \pm 2.39$ & $42.13 \pm 2.89$ & 0.782 \\
\hline Age range (years) & $21-58$ & $17-62$ & \\
\hline \multicolumn{4}{|l|}{ Diagnosis } \\
\hline AML & 6 & 8 & 0.839 \\
\hline Non-Hodgkin's lymphoma & 4 & 7 & \\
\hline Myelodysplastic syndrome & 2 & 2 & \\
\hline Myeloma & 1 & 4 & \\
\hline Others & 3 & 3 & \\
\hline \multicolumn{4}{|l|}{ Donor type } \\
\hline HLA-identical siblings & 11 & 13 & 0.037 \\
\hline Matched unrelated donors & 4 & 2 & \\
\hline Autologous & 1 & 9 & \\
\hline \multicolumn{4}{|l|}{ Source of hematopoietic stem cells } \\
\hline $\mathrm{BM}$ & 11 & 12 & 0.332 \\
\hline Peripheral blood & 5 & 12 & \\
\hline \multicolumn{4}{|l|}{ Conditioning } \\
\hline $\mathrm{BU}-\mathrm{CY}$ & 10 & 10 & 0.289 \\
\hline BU-BCNU-etoposide & 2 & 6 & \\
\hline CY-TBI & 3 & 2 & \\
\hline Melphalan & 1 & 5 & \\
\hline Others & 0 & 1 & \\
\hline Gut GVHDa & $5 / 15$ & $2 / 15$ & 0.39 \\
\hline Overall GVHD $(\text { grade } \geqslant 2)^{\mathrm{a}}$ & $7 / 15$ & $3 / 15$ & 0.245 \\
\hline \multicolumn{4}{|l|}{ Patterns of $B K V$ viruria } \\
\hline Persistent and peaking & 11 & 6 & $<0.001$ \\
\hline Persistent & 4 & 1 & \\
\hline Transient & 0 & 3 & \\
\hline Negative & 1 & 14 & \\
\hline Hemorrhagic cystitis (grade $\geqslant 2$ ) & 3 & 0 & 0.057 \\
\hline
\end{tabular}

Abbreviation: $\mathrm{BCNU}=$ carmustine.

${ }^{\mathrm{a}}$ Applicable only to patients receiving allogeneic HSCT.

fecal BKV load is typical of what might be expected of in reactivation of latent viruses after organ transplantation. ${ }^{13}$

Although the proposition of the gastrointestinal tract as a BKV latency site would need further validation in patients and normal people, it may provide valuable insights into BKV epidemiology. The seroprevalence of BKV increases from about $50 \%$ in children to $70-90 \%$ in adults. ${ }^{14}$ The high BKV seroprevalence has never been adequately explained, assuming urine to be the only means of transmission. A fecal-oral route, however, offers an additional epidemiologic explanation for the high viral prevalence.

Data on urinary BKV excretion in this study confirmed once again the consistent link between allogeneic HSCT, GVHD, significant BKV viruria, and severe postengraftment HC. ${ }^{3,6,8,15}$ We have previously postulated that immunosuppression allowing unchecked viral replication, in combination with the subsequent allogeneic reactivity against viral antigens upon hematopoietic engraftment (which might be heightened by GVHD) leads to severe HC. 
In this study, however, we were unable to observe any apparent association between fecal BKV excretion and gut GVHD. Nevertheless, this study contained a relatively small number of patients, so that the potential relationship between fecal BKV excretion and gut GVHD would need further examination in larger number of HSCT recipients.

Our observations provide two positive leads for future studies. Firstly, the factors predisposing to, and the biologic and clinical significance of, BKV latency in the gastrointestinal tract should be evaluated. In particular, recent study has identified BKV DNA sequence from colorectal cancers, suggesting that this virus may have a function in the pathogenesis of these diseases. ${ }^{12}$ Secondly, 8 out of 40 patients $(20 \%)$ demonstrated significant fecal BKV excretion before BM conditioning. This was lower than that of $40 \%$ reported from stool and rectal swab samples in hospitalized children, based on the detection of the BKV $T$-antigen gene. ${ }^{5}$ Whether this reflects an age-related difference in BKV fecal excretion and hence differences in viral epidemiology in health and disease or a technical difference in BKV DNA detection remains to be determined.

\section{Acknowledgements}

We thank Dr Albert KW Lie, Professor Raymond Liang and the nursing staff of the Bone Marrow Transplantation in Queen Mary Hospital, Hong Kong, for their expert management of patients recruited in this study. We are also grateful to Dr Daniel YT Fong, assistant professor, Department of Nursing Studies, University of Hong Kong, for his expert advice in statistical analysis.

\section{References}

1 Dörries K. Human polyomaviruses. In: Zuckerman AJ, Banatvala JE, Pattison JR (eds). Principles and Practice of Clinical Virology. 4th edn John Wiley \& Sons: New York, 2000 pp 619-643.

2 Nickeleit V, Klimkait T, Binet IF, Dalquen P, Del Zenero V, Thiel $\mathrm{G}$ et al. Testing for polyomavirus type BK DNA in plasma to identify renal-allograft recipients with viral nephropathy. N Engl J Med 2000; 342: 1309-1315.
3 Leung AY, Yuen KY, Kwong YL. Polyoma BK virus and haemorrhagic cystitis in haematopoietic stem cell transplantation: a changing paradigm. Bone Marrow Transplant 2005; 36: 929-937.

4 Ricciardiello L, Laghi L, Ramamirtham P, Chang CL, Chang DK, Randolph AE et al. JC virus DNA sequences are frequently present in the human upper and lower gastrointestinal tract. Gastroenterology 2000; 119: 1228-1235.

5 Vanchiere JA, Nicome RK, Greer JM, Demmler GJ, Butel JS. Frequent detection of polyomaviruses in stool samples from hospitalized children. J Infect Dis 2005; 192: 658-664.

6 Leung AY, Suen CK, Lie AK, Liang RH, Yuen KY, Kwong YL. Quantification of polyoma BK viruria in hemorrhagic cystitis complicating bone marrow transplantation. Blood 2001; 98: 1971-1978.

7 Hung IF, Cheng VC, Wu AK, Tang BS, Chan KH, Chu CM et al. Viral loads in clinical specimens and SARS manifestations. Emerg Infect Dis 2004; 10: 1550-1557.

8 Wong AS, Chan KH, Cheng VC, Yuen KY, Kwong YL, Leung AY. Relationship of pretransplantation polyoma BK virus serologic findings and BK viral reactivation after hematopoietic stem cell transplantation. Clin Infect Dis 2007; 44: $830-837$.

9 Hirsch HH. BK virus: opportunity makes a pathogen. Clin Infect Dis 2005; 41: 354-360.

10 Pavlakis M, Haririan A, Klassen DK. BK virus infection after non-renal transplantation. Adv Exp Med Biol 2006; 577: 185-189.

11 Hirsch HH, Drachenberg CB, Steiger J, Ramos E. Polyomavirus-associated nephropathy in renal transplantation: critical issues of screening and management. Adv Exp Med Biol 2006; 577: $160-173$.

12 Casini B, Borgese L, Del Nonno F, Galati G, Izzo L, Caputo $\mathrm{M}$ et al. Presence and incidence of DNA sequences of human polyomaviruses $\mathrm{BKV}$ and $\mathrm{JCV}$ in colorectal tumor tissues. Anticancer Res 2005; 25: 1079-1085.

13 Funk GA, Gosert R, Hirsch HH. Viral dynamics in transplant patients: implications for disease. Lancet Infect Dis 2007; 7: 460-472.

14 Stolt A, Sasnauskas K, Koskela P, Lehtinen M, Dillner J. Seroepidemiology of the human polyomaviruses. J Gen Virol 2003; 84: 1499-1504.

15 Bogdanovic G, Priftakis P, Giraud G, Kuzniar M, Ferraldeschi R, Kokhaei $\mathrm{P}$ et al. Association between a high BK virus load in urine samples of patients with graft-versus-host disease and development of hemorrhagic cystitis after hematopoietic stem cell transplantation. J Clin Microbiol 2004; 42 : 5394-5396. 